\title{
Photoallergic reaction in a patient receiving vandetanib for metastatic follicular thyroid carcinoma: a case report
}

\author{
Jennifer Goldstein ${ }^{1}$, Anisha B. Patel ${ }^{2}$, Jonathan L. Curry ${ }^{3}$, Vivek Subbiah ${ }^{4}$ and Sarina Piha-Paul ${ }^{4^{*}}$
}

\begin{abstract}
Background: Novel targeted agents have been increasingly developed and tested in clinical trials over the past 5-10 years, many with unknown and unanticipated side effects. We describe here a case of a patient with a history of metastatic follicular thyroid carcinoma that we believe developed vandetanib-associated photoallergic dermatitis while enrolled on a phase 1 clinical trial.

Case presentation: A 51-year-old Caucasian female with poorly differentiated, metastatic follicular thyroid carcinoma presented with a cutaneous eruption that developed over 3 to 4 days while treated on phase 1 clinical trial with vandetanib-based therapy. Given the concern for photoallergic dermatitis, vandetanib was discontinued and supportive care provided including topical and oral steroid administration. Her cutaneous eruption improved and she was successfully re-challenged with vandetanib.

Conclusion: Tyrosine kinase inhibitors, such as typo-vandetinib, with various therapeutic targets have come to the forefront of oncologic therapy in recent years. It is important to have a better understanding of the side effect profile and management in order to anticipate adverse events and maintain patient safety in future clinical trials.
\end{abstract}

Keywords: Photoallergic reaction, Vandetanib, Metastatic follicular thyroid carcinoma, Tyrosine kinase inhibitor, Investigational cancer therapeutics

\section{Background}

Novel targeted agents have been increasingly developed and tested in clinical trials over the past $5-10$ years. In clinical trials, drug reactions cannot always be anticipated and novel side effects can be encountered. Vandetinib is a multikinase inhibitor. Herein, we describe a patient with metastatic follicular thyroid carcinoma who we believe developed vandetanib-associated photoallergic dermatitis while enrolled on a phase 1 clinical trial.

\section{Case presentation}

A 51-year-old female with poorly differentiated, metastatic follicular thyroid carcinoma presented with a cutaneous eruption that developed over 3 to 4 days. One month prior to the development of the rash, she had

\footnotetext{
* Correspondence: spihapau@mdanderson.org

${ }^{4}$ Department of Investigational Cancer Therapeutics, University of Texas MD Anderson Cancer Center, 1515 Holcombe Boulevard, Unit 455, Houston TX 77030, USA

Full list of author information is available at the end of the article
}

begun therapy on a clinical trial with a combination of vandetanib at $300 \mathrm{mg}$ by mouth daily and everolimus, a mammalian target of rapamycin (mTOR) inhibitor, at $5 \mathrm{mg}$ by mouth daily (NCT01582191). She had a fiveyear history of thyroid cancer which had progressed despite thyroidectomy, radioactive iodine ablation therapy, chemotherapy, targeted therapy, radiation, and other novel agents. During follow up, her major complaint was new rash. The erythematous eczematous plaques started on the chest and posterior neck, with vesiculation of the posterior neck plaques one day after the rash was first noted. The lesions subsequently spread diffusely in sunexposed areas over the chest, the upper portion of the back of the neck, and the bilateral forearms, sparing the shoulders, abdomen, pelvis, and legs. Borders were welldemarcated adjacent to sun-protected areas (Figure 1). She described the rash as pruritic with desquamation. The patient denied pain or involvement of the mucous membranes. She reported heavy sun exposure approximately 2 weeks prior to the visit, but did use SPF 50 

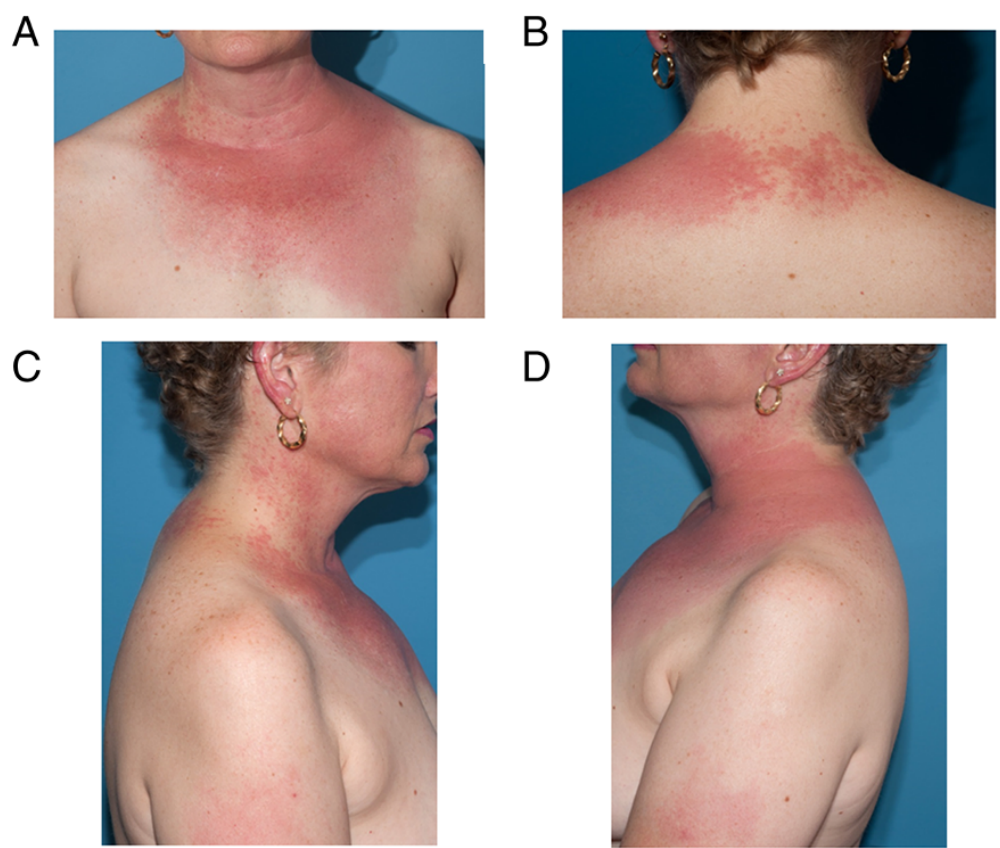

Figure 1 Dermatologic adverse events to vandetanib. A) Anterior chest. B) Upper portion of the back of the neck. C and D) Shoulders and arms showing sparing non-sun exposed areas.

sunscreen and wore long sleeves and long pants. However, she had, since that episode of heavy sun exposure, daily sun exposure without use of sunscreen. She did not report any new medications or changes to her current regimen. She held the study drugs for 1 day prior to the visit but otherwise was $100 \%$ compliant over the past month. Due to Grade 3 skin rash, the patient stopped the vandetanib and everolimus after being seen in clinic.

The patient was prescribed a $4 \mathrm{mg}$ methylprednisolone dose pack, hydroxyzine for itching, clobetasol shampoo, triamcinolone $0.1 \%$ cream and an antibiotic to prevent superinfection. Aggressive photoprotection was also recommended.

Subsequent dermatology evaluation revealed postinflammatory erythema with few areas of eczematous dermatitis remaining. Photoallergic dermatitis was suspected. A $4 \mathrm{~mm}$ punch biopsy showed superficial perivascular dermatitis with eosinophils and focal spongiosis. Histologic features were consistent with a reaction to an internal antigen, such as a medication leading to photo allergic reaction (Figure 2). Based on the timing of the rash 2 weeks after the initial severe sun exposure, the photodistribution of the rash, history of vesiculation and pruritus, and the histologic features, the patient was diagnosed with photoallergic dermatitis. Laboratory results included a normal complete blood count and comprehensive metabolic panel.

Dermatology placed the patient on an oral prednisone taper. Vandetanib continued to be held. After an additional week, the patient noted decreased erythema and no further blistering. There were no new areas of involvement, but she continued to have rare eczematous plaques that may have been post inflammatory erythema.

The patient was re-challenged with vandetanib two weeks after resolution of the rash after completion of the steroid taper and with institution of strict photoprotection. The rash did not return and the patient is tolerating the study drug well. She continues to follow-up with the phase 1 clinic.

\section{Conclusions}

Tyrosine kinase inhibitors, with various therapeutic targets, have come to the forefront of oncologic therapy in recent years. With block buster drugs such as imatinib for chronic myelogenous leukemia and gastrointestinal stromal tumor and vemurafenib for melanoma, drug companies and academic centers have formed collaborations to develop these types of agents in multiple tumor types. Vandetanib, an oral tyrosine kinase inhibitor, targets vascular endothelial growth factor receptor 2, the epidermal growth factor receptor, and RET [1,2]. It is approved by the United States Food and Drug Administration and European Medicines Agency for the treatment of advanced medullary thyroid cancer. Most recently, trials with vandetanib have also been conducted in hepatocellular carcinoma, and non-small cell lung cancer [3].

Tyrosine kinase inhibitors are known to have side effects including nontrivial dermatologic toxicity, usually manifesting as acneiform eruption, xerosis, eczema and 


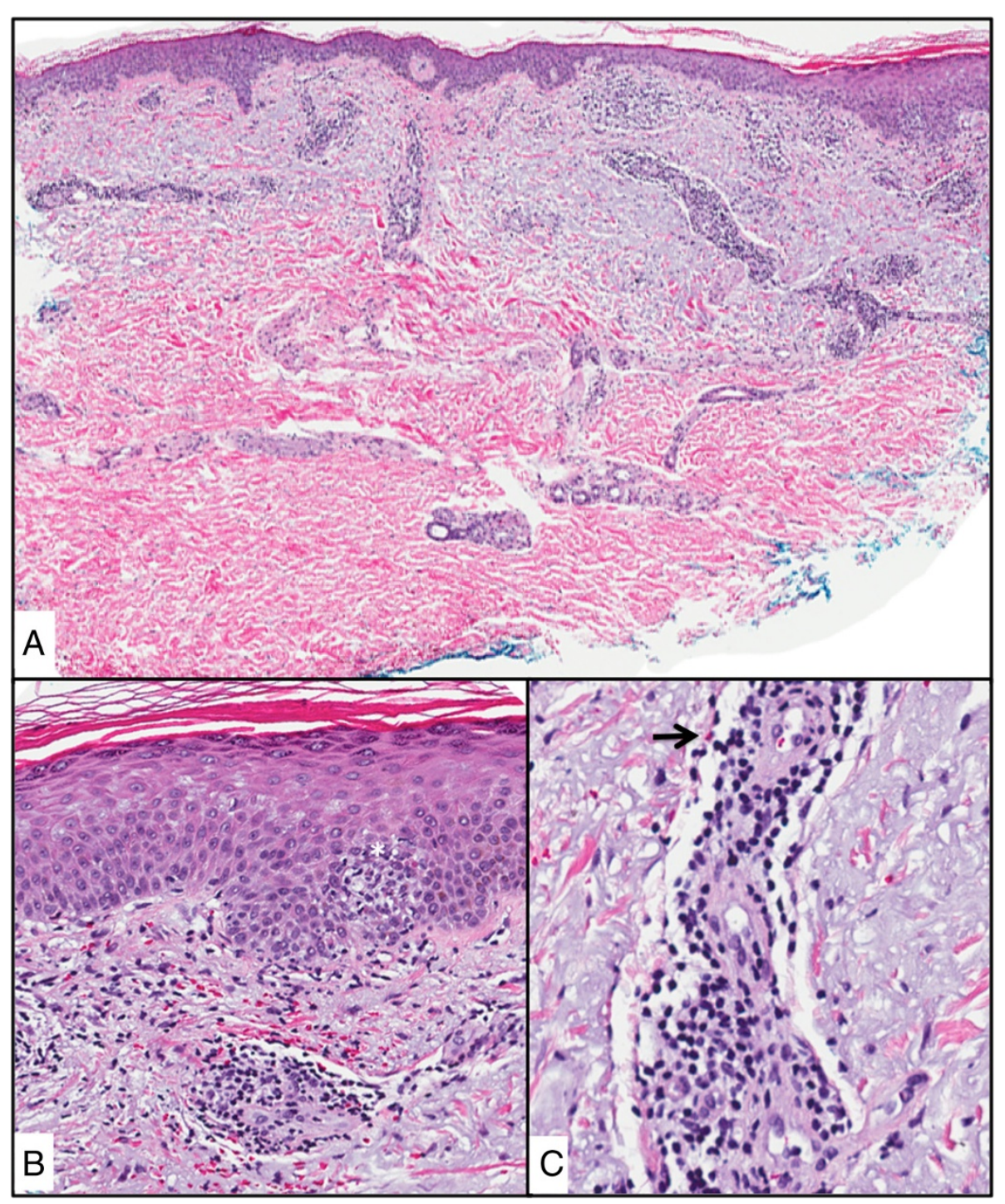

Figure 2 Hematoxolin and eosin (H\&E) A) skin punch with superficial and deep perivascular lymphocytic infiltrate and epidermal spongiosis, B) epidermal spongiosis with exocytosis of lymphocytes $\left(^{*}\right.$ C) perivascular lymphocytes with eosinophils (arrow).

cutaneous epithelial proliferations (e.g. actinic keratosis, keratoacanthoma, squamous cell carcinomas), with grade $1-2$ rashes reportedly occurring in $11-82 \%$ of patients [4]. Photodistributed reactions rarely occur and can be divided into phototoxic or photoallergic etiologies [5]. Phototoxic reactions occur because of the damaging effects of lightactivated compounds on cell membranes and, in some instances, DNA [5,6]. Photoallergic reactions are cellmediated immune responses to a light-activated compound [5]. Phototoxic reactions develop in most individuals if they are exposed to sufficient amounts of light and drug, and develop within days of ultraviolet (UV) exposure [5]. Photoallergic reactions usually take days to weeks to develop as the immune response develops [5]. Phototoxic

Table 1 Photosensitivity reactions to vandetanib reported in the literature

\begin{tabular}{lllllll}
\hline First author, year (Ref.) & Trial design & Vandetanib arm & Control arm & Enrollment no. & Sample size & Underlying cancer \\
\hline Katsuyuki Kiura, 2008 [9] & Phase Ila & $100-300$ mg/day & N/A & 53 & 12 & NSCLC \\
Heidi Kong, 2009 [11] & N/A & N/A & N/A & N/A & 1 & Recurrent brain tumor \\
Chih-Hsiang Chang, 2009 [10] & Phase II & 300 mg/day & N/A & N/A & 1 & HCC \\
Damien Giacchero, 2012 [12] & Phase II/II & $300 \mathrm{mg} /$ day & Placebo & 63 & 23 & Metastatic thyroid cancer \\
\hline
\end{tabular}


reactions occur more commonly than photoallergic reactions. In our case, an eruption appeared 2 weeks after the intense sun exposure, the lesions were pruritic, and limited to sun exposed areas therefore based on clinical presentation was more likely a photoallergic rather than phototoxic rash.

In the literature, few cases of photosensitivity reaction to vandetanib have been described in detail. However, it is important to note that in clinical trials with vandetanib, rash is commonly listed as a side effect although without specific detail given [7-9]. In one trial, up to grade 2 rash developed in 12 of $46(26 \%)$ patients using vandetanib for metastatic breast cancer [8]. In other cases more severe reactions have been reported. For example, Chang et al. reports a case of a 60-year-old man with hepatocellular carcinoma treated on trial with vandetanib who developed bullous lesions related to sun exposure [10]. Kong et al. reports two cases of phototoxicity induced hyperpigmentation [11]. Giacherro reports 21 of 63 (33\%) patients developed erythematous skin eruptions ranging from exaggerated sunburn after moderate sun exposure to a severe photodistributed erythematous eruption associated with desquamation and pruritus [12]. These trials findings are summarized in Table 1.

Photosensitizing chemicals usually have a low molecular weight (200 to $500 \mathrm{Da}$ ), are planar, tricyclic, or polycyclic configurations and often contain heteroatoms that enable resonance stabilization $[6,10]$. They absorb UV light, a characteristic that is essential to be regarded as a photosensitizer. Vandetanib is a low-molecular weight molecule with a polycyclic structure $[6,10]$. Thus, it is plausible that vandetanib might be able to induce photosensitivity $[6,10]$. It also appears the degree of sun exposure does correlate with the severity of the rash [13].

In this patient, it is also important to consider photo accentuation of a typical drug eruption or other nonspecific common polymorphous skin reactions found in many patients on experimental agents. In this case, however, the eruption was thought to be most consistent with a photoallergic reaction as the patient's lesions were limited to photo-distributed areas (including sharp borders adjacent to sun-protected skin), the delayed onset of the eruption after the sun exposure and drug exposure, and the negative re-challenge when the patient was exercising strict photoprotection. The histology further supported a photoallergic versus phototoxic reaction as did the delayed onset of the eruption.

There are no defined guidelines as to the safety of rechallenge in patients who experience severe photoinduced reaction with vandetanib. It is been reported that these reactions usually resolve with the use of sunscreen and avoidance of sun exposure. In our case, holding the drug and giving both topical and oral steroids resulted in clearance of the rash. Ultimately, vandetanib was resumed with strict photoprotection and the patient tolerated continuance of therapy without further issues.

In conclusion, treatment with vandetanib and similar agents, either alone or in combination, may result in photodistributed rashes including photoallergic reactions. We hope to expand on the knowledge of skin reactions with the use of vandetanib to aid future researchers.

\section{Consent}

Written informed consent was obtained from the patient for publication of this Case report and any accompanying images. A copy of the written consent is available for review by the Editor of this journal.

\section{Abbreviations}

mTOR: Mammalian target of rapamycin; UV: Ultraviolet.

\section{Competing interests}

The authors declare that they have no competing interests.

\section{Authors' contributions}

All authors read and approved the final manuscript. JG participated in the study concept and design, analysis of the case, drafting of the manuscript and critical revision of the manuscript and important intellectual content. SP participated in the study concept and design, analysis of the case, drafting of the manuscript and critical revision of the manuscript and important intellectual content, and study supervision. AP participated in the study concept and design, analysis of the case, critical revision of the manuscript and important intellectual content, and provided technical support. JC participated in the study concept and design, analysis of the case, critical revision of the manuscript and important intellectual content, and provided technical support. VS participated in the study concept and design, analysis of the case, drafting of the manuscript and critical revision of the manuscript and important intellectual content.

\section{Acknowledgements}

We are indebted to Margaret Brown and Cristel Bastida.

\section{Author details}

${ }^{1}$ Division of Cancer Medicine, Medical Oncology and Hematology Fellowship Program, University of Texas MD Anderson Cancer Center, 1515 Holcombe Boulevard, Houston, TX 77030, USA. ²Department of Dermatology, University of Texas MD Anderson Cancer Center, 1515 Holcombe Boulevard, Houston, TX 77030, USA. ${ }^{3}$ Department of Pathology, University of Texas MD Anderson Cancer Center, 1515 Holcombe Boulevard, Houston, TX 77030, USA.

${ }^{4}$ Department of Investigational Cancer Therapeutics, University of Texas MD Anderson Cancer Center, 1515 Holcombe Boulevard, Unit 455, Houston TX 77030, USA.

Received: 7 October 2014 Accepted: 21 January 2015

Published online: 13 February 2015

\section{References}

1. Wedge SR, Ogilvie DJ, Dukes M, Kendrew J, Chester R, Jackson JA, et al. ZD6474 inhibits vascular endothelial growth factor signaling, angiogenesis, and tumor growth following oral administration. Cancer Res. 2002;62(16):4645-55.

2. Carlomagno F, Vitagliano D, Guida T, Ciardiello F, Tortora G, Vecchio G, et al. ZD6474, an orally available inhibitor of KDR tyrosine kinase activity, efficiently blocks oncogenic RET kinases. Cancer Res. 2002;62(24):7284-90.

3. Heymach JV. ZD6474-clinical experience to date. Br J Cancer. 2005;92 Suppl 1:S14-20.

4. Huang X, Patel S, Ahmed N, Seiter K, Liu D. Severe toxicity of skin rash, fever and diarrhea associated with imatinib: case report and review of skin toxicities associated with tyrosine kinase inhibitors. Drug Des Devel Ther. 2009;2:215-9. 
5. Stein KR, Scheinfeld NS. Drug-induced photoallergic and phototoxic reactions. Expert Opin Drug Saf. 2007;6(4):431-43.

6. Moore DE. Drug-induced cutaneous photosensitivity: incidence, mechanism, prevention and management. Drug Saf. 2002;25(5):345-72.

7. Rosen AC, Wu S, Damse A, Sherman E, Lacouture ME. Risk of rash in cancer patients treated with vandetanib: systematic review and meta-analysis. J Clin Endocrinol Metab. 2012;97(4):1125-33.

8. Miller KD, Trigo JM, Wheeler C, Barge A, Rowbottom J, Sledge G, et al. A multicenter phase II trial of ZD6474, a vascular endothelial growth factor receptor-2 and epidermal growth factor receptor tyrosine kinase inhibitor, in patients with previously treated metastatic breast cancer. Clin Cancer Res. 2005;11(9):3369-76.

9. Kiura K, Nakagawa K, Shinkai T, Eguchi K, Ohe Y, Yamamoto N, et al. A randomized, double-blind, phase lla dose-finding study of Vandetanib (ZD6474) in Japanese patients with non-small cell lung cancer. J Thorac Oncol. 2008;3(4):386-93.

10. Chang $\mathrm{CH}$, Chang JW, Hui $\mathrm{CY}$, Yang $\mathrm{CH}$. Severe photosensitivity reaction to vandetanib. J Clin Oncol. 2009;27(27):e114-5.

11. Kong HH, Fine HA, Stern JB, Turner ML. Cutaneous pigmentation after photosensitivity induced by vandetanib therapy. Arch Dermatol. 2009;145(8):923-5.

12. Giacchero D, Ramacciotti C, Arnault JP, Brassard M, Baudin E, Maksimovic L, et al. A new spectrum of skin toxic effects associated with the multikinase inhibitor vandetanib. Arch Dermatol. 2012;148(12):1418-20.

13. Fava P, Quaglino P, Fierro MT, Novelli M, Bernengo MG. Therapeutic hotline. A rare vandetanib-induced photo-allergic drug eruption. Dermatol Ther. 2010;23(5):553-5.

\section{Submit your next manuscript to BioMed Central and take full advantage of:}

- Convenient online submission

- Thorough peer review

- No space constraints or color figure charges

- Immediate publication on acceptance

- Inclusion in PubMed, CAS, Scopus and Google Scholar

- Research which is freely available for redistribution 